\section{A three-valued photoelectrochemical logic device realising accept anything and consensus operations $\dagger$}

Received 15th December 2014

Accepted 20th January 2015

DOI: $10.1039 / c 4 c c 09980 j$

www.rsc.org/chemcomm

\author{
M. Warzecha, ${ }^{a}$ M. Oszajca, ${ }^{b}$ K. Pilarczyk* ${ }^{* d}$ and K. Szaciłowski*de
}

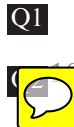

A new application of a hybrid material exhibiting the photoelectrochemical photocurrent switching (peps) effect in a three-valued logic device is reported. In contrast to other similar peps-based systems, the one described here is capable of performing basic ternary logic operations: gullibility and consensus.

The research in molecular-scale logic devices has already reached a certain level of maturity. This odyssey started with the seminal Nature paper by A. P. de Silva ${ }^{1}$ and within twenty years evolved into a rapidly developing field. There are hundreds of examples of all possible logic gates and switches, as well as arithmetic circuits, multiplexers and demultiplexers, encoders and even simple cryptographic devices. ${ }^{2-7}$ Nonetheless, two fields, whose roles are crucial for information processing, have not been explored so far. One of them is reversible logic. The molecular-scale reversible logic gate, the so called Feynman gate was reported only once by U. Pischel in $2009 .{ }^{8}$ The other concept, which did not arouse sufficient interest, is that of multivalued and fuzzy logic. Although, these approaches have already been implemented in molecular-scale devices, they are capable of operating only in solutions: three-valued logic by U. Pischel ${ }^{8}$ and fuzzy logic by P. L. Gentili. ${ }^{9-14}$ The first attempt towards the introduction of ternary logic in solid state systems utilising the photoelectrochemical photocurrent switching effect (the PEPS effect) was presented in 2011 by Oszajca $e t a l .{ }^{15}$ The system worked in an aqueous electrolyte,

${ }^{a}$ Strathclyde Institute of Pharmacy and Biomedical Sciences,

University of Strathclyde, 161 Cathedral Street, Glasgow, UK

${ }^{b}$ Department of Chemistry and Applied Biosciences, ETH Zurich,

Vladimir-Prelog-Weg 1, 8093 Zurich, Switzerland

$50{ }^{c}$ AGH University of Science and Technology, Faculty of Physics and Applied Computer Science, al. A. Mickiewicza 30, 30-059 Kraków, Poland. E-mail: kacper.pilarzyk@fis.agh.edu.pl

${ }^{d}$ AGH University of Science and Techno Academic Centre for Materials and Nanotechnology, al. A. Mickiewicza 30, 30-059 Kraków, Poland

${ }^{e}$ AGH University of Science and Technology, Faculty of Non-Ferrous Metals, al. A. Mickiewicza 30,30-059 Kraków, Poland. E-mail: szacilow@agh.edu.pl

$\dagger$ Electronic supplementary information (ESI) available. See DOI: 10.1039/c4cc09980j like almost all PEPS-based devices and generated photocurrent pulses in response to the optical stimulation, but its function could be only described as a three-state switch or a binary-toternary converter. This communication presents the first case of Boolean ternary logic operation performed in the photoelectrochemical device.

Wide band gap semiconductors, including titanium dioxide, generate photocurrent upon excitation with light of appropriate wavelength. Its direction (polarity) depends on the doping state of the semiconductor: n-type semiconductors generate typically anodic photocurrents, whereas the p-type semiconductors generate the cathodic ones. However, when the surface of semiconductor is modified with the molecular species exhibiting significant light absorption (usually at the lower energies than the band gap width) they may inject electrons into the conduction band, or holes into the valence band of the semiconductor. This process, called photosensitization, is commonly utilised in dye-sensitized solar cells. If the surface molecule is also redoxactive and an appropriate sacrificial reagent is present in the solution, the process of photosensitization becomes redoxcontrolled, which gives rise to various switching phenomena. ${ }^{16}$ These effects can be used in the implementation of numerous binary logic functionalities. ${ }^{17,18}$

Here we report the use of certain photoelectrochemical properties of titanium dioxide modified with hexacyanobutadienide (1) logic device. The parent compounds, prepared using a wellestablished synthetic route from tetracyanoethene, undergo strong
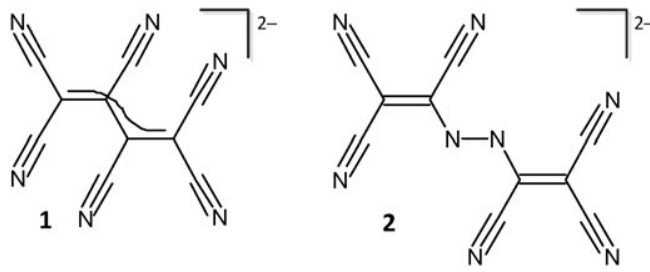

Scheme 1 The structures of cyanocarbon modifiers. and hexacyanodiazahexadienide (2) anions (Scheme 1) in a ternary 
1 adsorption at the surface of n-type titanium dioxide with subsequent reduction by conduction band electrons, originating from oxygen vacancies. Such a process, which involves the withdrawal of charge carriers from the semiconductor is usually called surface transfer doping ${ }^{19}$ and has been recently observed in chromate(vi) and fluorochromate(vi)-modified titania. ${ }^{20}$

The spectroscopic measurements indicate that the modifiers are adsorbed in the anionic form, and a new absorption band ranging from 2.4 to $3.0 \mathrm{eV}$ can be observed. This transition can be associated with a charge transfer process involving surface Ti(Iv) centres and adsorbed cyanocarbon anions.

The photoelectrochemical data suggest that novel materials show only minor photosensitization with photocurrents generated with the excitation wavelength from 300 to $525 \mathrm{~nm}$. Noneeless, the photocurrent switching effect is strongly pronounced. The anodic photocurrents are recorded only within a 300-400 nm window and at the positive potential of the photoelectrode, which is a typical behaviour observed for titanium dioxide. At the same time, the change of the polarization leads to the generation of cathodic photocurrents - at first only within the $400-500 \mathrm{~nm}$ range (together with the anodic photocurrent) but at the lower photoelectrode potentials only the cathodic photocurrents are observed (Fig. 1).

This peculiar behaviour (quite different from that of other PEPS-based systems) can be explained by the presence of two independent photocurrent generators. The inner parts of the $\mathrm{TiO}_{2}$ nanoparticles retain their n-type character and are responsible for anodic photocurrent generation. On the other hand, the surface transfer doping with 1 or 2 results in a significant decrease in the conduction band electrons concentration, which is reflected by a strong increase of the band bending at the interface, and contributes to the widening of the depletion layer at the outer part of the particles (Fig. S1 and S2, ESI $\dagger$ ). Such a mechanism is consistent with the observed photocurrent profile and is also supported by the data published on strong electron acceptors adsorbed at n-type semiconductors, where surface hydroxyl ions as well as oxygen vacancies can serve as electron donors. ${ }^{21-23}$ In the case of cyanomethylene

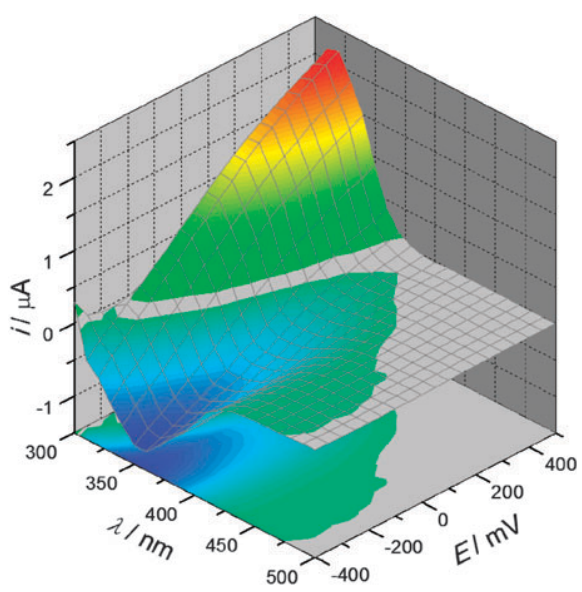

Fig. 1 The photocurrent action spectra recorded for $\mathrm{TiO}_{2}$ modified with 1 in the presence of oxygen in $0.1 \mathrm{M} \mathrm{KNO}_{3}$ (cf. Fig. S3 (ESI $\dagger$ ) for compound 2).

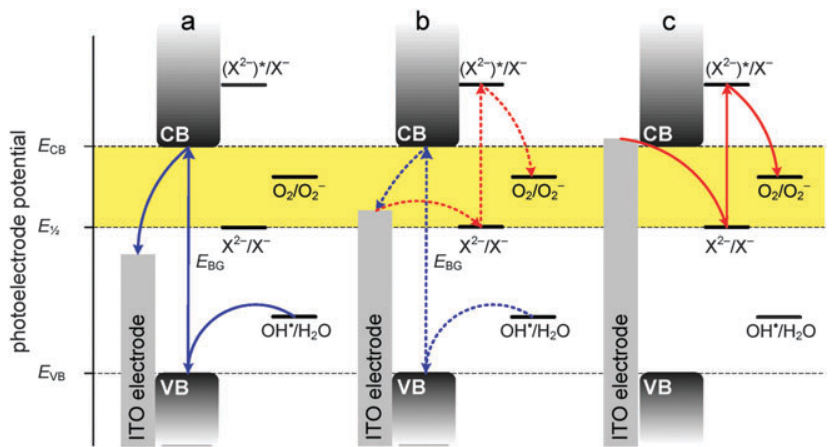

1

Fig. 2 The mechanism of the photocurrent generation under anodic (a), mixed (b) and cathodic conditions (c). X stands for the surface molecule.

compounds the obtained surface species behave as efficient chromophores, absorbing in the visible light region, which can be involved in the photoinduced electron transfer processes. Therefore $\mathrm{TiO}_{2}$ with surface modified with 1 or 2 can be regarded as a hybrid material combining some features of n-type and p-type semiconductors characterised by significantly different band gaps (Fig. 2).

At the potentials higher than the switching potential, according to the Butler-Volmer equation, the p-type semiconductor cannot generate any photocurrent. The same situation occurs with the more negative polarization and anodic photocurrents. Hence, one may conclude that a subtle competition between two processes (Fig. 2b) governs the overall polarity of the photocurrent generated at the modified electrodes and that in the intermediate state no net photocurrent is generated (which corresponds to the unknown state of the device). Furthermore, different surface states induce fine variations of surface molecules redox properties, leading to slightly different frontier orbital energies; hence the wavelength dependence is observed.

It can be noticed that the photocurrent action map can be divided into three distinct regions according to the photocurrent polarity and intensity: anodic (with dominating anodic photocurrent), cathodic (with dominating cathodic photocurrent) and null photocurrent area, where anodic and cathodic photocurrents effectively compensate each other.

It appears immediately that such a behaviour corresponds to a three-state logic circuit with $-1,0$ and 1 (FALSE, UNKNOWN, TRUE) outputs in a very natural way: positive (anodic) photocurrent corresponds to logical " 1 ", negative (cathodic) photocurrent to logical "-1", whereas areas with no net photocurrent to logical “0”. Furthermore, individual ranges of the applied potential and the incident light wavelength can be assigned to ternary Boolean values, as indicated in Fig. 3 ( $c f$. Fig. S4 (ESI $\dagger$ ) for compound 2).

A simple analysis leads to the conclusion that the photoelectrodes composed of cyanocarbon-modified titanium dioxide behave like ternary (three-valued) logic devices. These devices (and their functions) may be described in terms of one of the basic operators - the accept anything (or gullibility) operator or consensus operator 

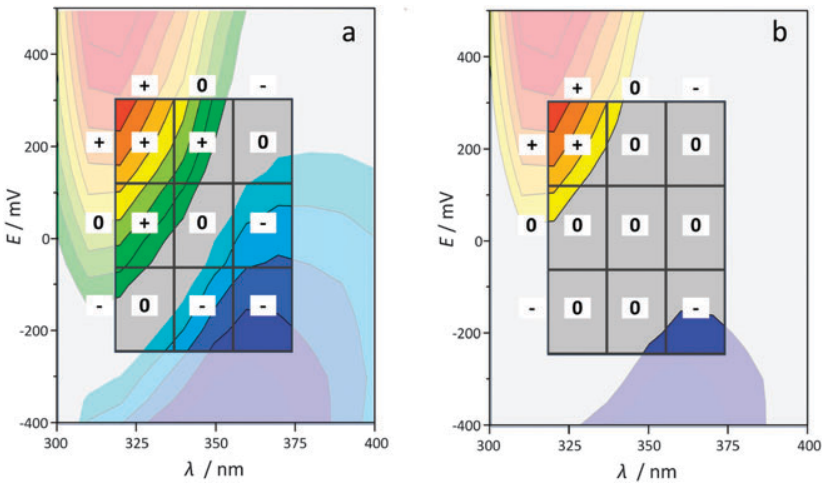

Fig. 3 A fragment of a photocurrent action map with the three-valued truth table corresponding to the accept anything operation (a). The consensus operation can be realized by neglecting the photocurrents with lowest intensities (b) a) Input a

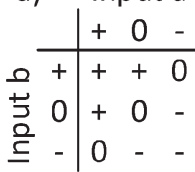

b) Input a

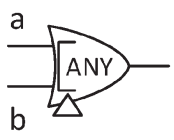

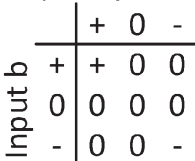

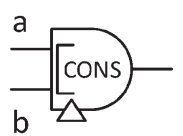

Fig. 4 The truth tables and symbols for the ternary gullibility (a) and consensus (b) operations.

The gullibility operator returns UNKNOWN output either with two UNKNOWN inputs or when two inputs are of opposite character (one TRUE and one FALSE). In other cases it yields an output equal to any non-UNKNOWN input. The truth table presented (Fig. 4a) exactly matches the one which is proposed in photocurrent action map (Fig. 3a).

A simple modification of the photocurrent threshold, which neglects some isovalue lines adjoining the UNKNOWN area leads to another ternary basic operator - consensus. The consensus operation applied to two ternary variables returns FALSE if both inputs are FALSE, TRUE if both are TRUE or UNKNOWN in all the other cases. These two gates of a dual nature are natural extensions of OR and AND gates in three-valued logic. negation operation (the ternary analogue of NOT); hence a complete set of logic operators cannot be achieved.

The consensus and gullibility operations are rarely used in the design of ternary logic circuits but are extremely useful in the ternary logic. Moreover, contrary to the molecular logic gates operating in solution, the presented system can be easily concatenated. ${ }^{25}$ The output (current) is compatible with one of the inputs (voltage) and an external resistor would be sufficient for the proper communication between individual gates. Similar solution can be used to prevent bidirectional information transfer which could lead to undesired feedback loop - a simple addition of a Schottky diode (e.g. based on a thin CdS layer $)^{25}$ would provide unidirectional information transfer. Since the system is based on the interaction with light (from an external source), the signal amplification is not required and the photocurrent amplitude may be sustained at the constant level within a circuit.

In the presented communication we show the application of nple hybrid materials (based on the cyanocarbon-modified Q hium dioxide) in the construction of ${ }^{\text {ree-valued photo- }}$ electrochemical logic devices, which rea he gullibility and consensus operations. This is the unique case, where two dual operators can be realised in the same chemical system just by a simple change in the output threshold values. We also propose the mechanism responsible for the properties of the investigated system along with the interpretation of the recorded photocurrent action spectra in terms of ternary Boolean logic. Such systems may become fundamental to multivalued optoelectronic logic circuits based on the easily accessible hybrid materials and may contribute to the development of this certainly underappreciated field of information processing.

Financial support from the National Science Centre (grant no. UMO-2011/03/B/ST5/01495) and the Foundation for Polish Science (grant no. 71/UD/SKILLS/2014) is gratefully acknowledged.

\section{Notes and references}

1 A. P. de Silva, H. Q. N. Gunaratne and C. P. McCoy, Nature, 1993, 364, 42-44.

2 U. Pischel, Angew. Chem., Int. Ed., 2007, 46, 4026-4040.

3 J. Andréasson and U. Pischel, Chem. Soc. Rev., 2010, 39, 174-188.

4 U. Pischel, Aust. J. Chem., 2010, 63, 148-164.

5 K. Szaciłowski, Chem. Rev., 2008, 108, 3481-3548.

6 K. Szaciłowski, Infochemistry. Information Processing at the Nanoscale, John Wiley \& Sons, Chichester, 2012.

7 A. P. De Silva, Molecular logic-based computation, Royal Chemical Society, Cambridge, 2012.

8 P. Remón, R. Ferreira, J. M. Montenegro, R. Suau, E. Pérez-Inestrosa and U. Pischel, ChemPhysChem, 2009, 10, 2004-2007.

9 P. L. Gentili, Chem. Phys., 2007, 336, 64-73.

10 P. L. Gentili, J. Phys. Chem. A, 2008, 112, 11992-11997.

11 P. L. Gentili, ChemPhysChem, 2011, 12, 739-745.

12 P. L. Gentili, Phys. Chem. Chem. Phys., 2011, 13, 20335-20344.

13 P. L. Gentili, RSC Adv., 2013, 3, 25523-25549.

14 P. L. Gentili, Dyes Pigm., 2014, 110, 235-248.

15 M. Oszajca, K. L. McCall, N. Robertson and K. Szaciłowski, J. Phys. Chem. C, 2011, 115, 12187-12195.

16 S. Gaweda, A. Podborska, W. Macyk and K. Szaciłowski, Nanoscale, 2009, 1, 299-316.

17 K. Szaciłowski and W. Macyk, Chimia, 2007, 61, 831-834.

18 A. Podborska, M. Oszajca, S. Gawda and K. Szaciłowski, IET Circ. Dev. Syst., 2011, 5, 103-114.

19 W. Chen, D. Qi, X. Gao and A. Tye Shen Wee, Prog. Surf. Sci., 2009, 84, 279-321.

20 J. Kuncewicz, P. Za̧bek, K. Kruczała, K. Szaciłowski and W. Macyk, J. Phys. Chem. C, 2012, 116, 21762-21770.

21 R. S. Davidson and R. M. Slater, J. Chem. Soc., Faraday Trans. 1, 1976, 76, 2416-2424.

22 R. Jono, J. Fujisawa, H. Segawa and K. Yamashita, J. Phys. Chem. Lett., 2011, 2, 1167-1170.

23 S. Manzhos, R. Jono, K. Yamashita, J. Fujisawa, M. Nagata and H. Segawa, J. Phys. Chem. C, 2011, 115, 21487-21493.

24 S.-C. Chen and M.-L. Shyu, Multimedia Data Engineering Applications and Processing, IGI Global, 2013.

25 J. Mech, R. Kowalik, A. Podborska, P. Kwolek and K. Szaciłowski, Aust. J. Chem., 2010, 63, 1330-1333.

\section{Q4}

